\title{
The Relative Roles of Diffusion and Uptake in Clearing Synaptically Released Glutamate Change during Early Postnatal Development
}

\author{
Christopher G. Thomas, Hua Tian, and Jeffrey S. Diamond \\ Synaptic Physiology Section, National Institute of Neurological Disorders and Stroke, National Institutes of Health, Bethesda, Maryland 20892-3701
}

Glutamate uptake by transporters expressed in astrocytes combines with synaptic structure to regulate the time that synaptically released glutamate remains in the extracellular space and, consequently, the duration and location of postsynaptic receptor activation. Both factors change greatly in the rodent hippocampus during the second postnatal week when most synapses become established and begin to mature, processes that are influenced by synaptically released glutamate. Transporter expression increases, potentially speeding removal of synaptically released glutamate, whereas extracellular space decreases, thereby slowing dilution. We investigated whether these competing changes influence the glutamate concentration time course and postsynaptic responses in the CA1 region of the mouse hippocampus during this critical period of synaptic development. Our results suggest that the glutamate concentration time course remains relatively consistent over this period, although the primary mechanisms regulating glutamate clearance change. Before the second postnatal week, clearance of synaptically released glutamate depends primarily on diffusion into large extracellular spaces, whereas later in development it relies more on increased uptake capacity. Thus, increased transporter expression during this period accompanies structural changes in the neuropil, preserving a relatively consistent glutamate concentration time course and ensuring that postsynaptic receptor activation remains brief and primarily localized to receptors close to release sites.

\section{Introduction}

Glutamate receptors mediate communication between neurons and facilitate synapse formation and maturation in the developing CNS (Adesnik et al., 2008; Bourne and Harris, 2008; Cline and Haas, 2008). In the CA1 region of the hippocampus, glutamate released from Schaffer collateral axons aids formation of nascent filopodia on pyramidal cell dendrites, conversion of motile filopodial to stable spines, and assembly of postsynaptic densities (Cohen-Cory, 2002; Bourne and Harris, 2008; Cline and Haas, 2008). Synaptic maturation is particularly rapid between postnatal day 7 (P7) and P10 (Li and Sheng, 2003). Factors influencing the clearance of synaptically released glutamate, including uptake by glutamate transporters and diffusion from synapses into surrounding extracellular spaces, change substantially during this period (Schmidt and Wolf, 1988; Bergles and Jahr, 1997; Danbolt, 2001; Syková and Nicholson, 2008), but the impact of these changes on synaptic transmission and maturation remains mostly unexplored.

In the mature hippocampus glial glutamate transporters GLAST (EAAT1) and GLT-1 (EAAT2) take up glutamate (Bergles and Jahr,

Received Nov. 3, 2010; revised Jan. 12, 2011; accepted Feb. 4, 2011.

This work was supported by the National Institute of Neurological Disorders and Stroke Intramural Research Program and the Human Frontier Science Program. We thank J.-H. Tao-Cheng, and Rita Azzam for help with electron microscopy, Carolyn Smith for help with confocal imaging, and S. Brian Andrews, William N. Grimes, William W. Kothmann, Nick Oesch, Syed Qadri, and Annalisa Scimemi for valuable discussions and helpful comments on this manuscript.

Correspondence should be addressed to Dr. Jeffrey S. Diamond, Building 35, Room 3C-1000, 35 Convent Drive, Bethesda, MD 20892-3701. E-mail: diamondj@ninds.nih.gov.

DOI:10.1523/JNEUROSCI.5953-10.2011

Copyright $\odot 2011$ the authors $\quad 0270-6474 / 11 / 314743-12 \$ 15.00 / 0$
1998; Tzingounis and Wadiche, 2007), thereby reducing the extent to which neurotransmitter activates synaptic and extrasynaptic receptors (Asztely et al., 1997; Arnth-Jensen et al., 2002; Scimemi et al., 2004). Glial transporter expression and glutamate uptake capacity is low in the neonatal ( $\mathrm{P} 0-\mathrm{P} 7)$ hippocampus but increases during synaptic maturation, reaching adult levels by P30 (Schmidt and Wolf, 1988; Ullensvang et al., 1997). Synaptically activated transporter currents recorded in hippocampal astrocytes are faster in adult $(>\mathrm{P} 60)$ than in juvenile (P12-P14) rats (Diamond, 2005), suggesting that developmental increases in transporter expression speed clearance of synaptically released glutamate and may restrict the spatial extent of postsynaptic receptor activation. Transporter currents are even slower in neonatal hippocampus (Bergles and Jahr, 1997), suggesting that glutamate diffuses farther early in development and may compromise the specificity of synapse formation and maturation.

The concentration profile of synaptically released glutamate also is influenced by the geometry of the neuropil (Syková and Nicholson, 2008). Narrow, tortuous extracellular spaces impede transmitter diffusion and slow dilution, thus enhancing postsynaptic receptor activation within and around the synaptic cleft. Ultrastructural and diffusion studies indicate that the extracellular volume fraction $(\alpha)$ decreases by more than one-half between the first postnatal week and adulthood (Syková and Nicholson, 2008). Theoretically, decreased $\alpha$ and increased transporter expression during early postnatal development could exert opposing influences on the spatial extent of receptor activation.

We tested this hypothesis by comparing neuropil morphology, glutamate transporter expression, glial uptake dynamics, and NMDA receptor (NMDAR) activation in the hippocampal 
CA1 region of neonatal (P4-P6) and juvenile (P13-P15) mice. Biochemical and electrophysiological experiments indicated that transporter expression and uptake capacity increase during this period, and electron microscopy confirmed that the extracellular volume fraction decreases by one-half, likely slowing dilution of synaptically released glutamate. Clearance of glutamate released from neonatal synapses is mediated primarily by diffusion, whereas clearance in the juvenile depends more on glial uptake. The relative activation of synaptic and extrasynaptic NMDARs remains stable over this period, suggesting that developmental increases in transporter expression compensate for structural changes that slow transmitter dilution.

\section{Materials and Methods}

Slice preparation. Acute hippocampal brain slices were made from neonatal (P4-P6) and juvenile (P13-P15) wild-type C57BL/6 mice (male or female), in accordance with National Institute of Neurological Disorders and Stroke Animal Care and Use Committee guidelines. Briefly, mice were decapitated and brains were removed, placed in ice-cold slicing solution, trimmed, and then glued onto a vibratome stage. Juvenile mice were decapitated after being anesthetized with isoflurane, whereas neonatal mice were decapitated without receiving anesthesia. Transverse hippocampal slices ( $300 \mu \mathrm{m}$ thick) were cut on a vibrating microtome (VT1000S; Leica) in cold $\left(4^{\circ} \mathrm{C}\right)$, continuously oxygenated $\left(95 \% \mathrm{O}_{2} / 5 \%\right.$ $\mathrm{CO}_{2}$ ) slicing solution containing the following (in $\mathrm{mM}$ ): $125 \mathrm{NaCl}, 2.5$ $\mathrm{KCl}, 1.25 \mathrm{NaH}_{2} \mathrm{PO}_{4}, 25$ glucose, $25 \mathrm{NaHCO}_{3}, 0.5 \mathrm{CaCl}_{2}, 5 \mathrm{MgCl}_{2} ; 320$ $\mathrm{mOsm}, \mathrm{pH}$ 7.4. Slices were collected in a submersion chamber filled with slicing solution, incubated at $34^{\circ} \mathrm{C}$ for $30 \mathrm{~min}$ and then kept at room temperature until transferred to the recording chamber. Slices were used for up to $5 \mathrm{~h}$ after preparation.

Electrophysiology. NMDAR-mediated EPSCs were recorded from CA1 pyramidal neurons visualized with infrared differential interference microscopy (Axioskop 2FS; Zeiss). Slices were placed in a heated $\left(34^{\circ} \mathrm{C}\right.$ ) recording chamber and superfused $(\sim 3 \mathrm{ml} / \mathrm{min})$ with artificial CSF containing the following (in $\mathrm{mM}$ ): $2.5 \mathrm{CaCl}_{2}, 1.3 \mathrm{MgCl}_{2}, 0.01 \mathrm{D}$-serine, and 0.1 picrotoxin to block $\mathrm{GABA}_{\mathrm{A}}$ receptors. Shaffer collaterals were cut between CA3 and CA1 regions to reduce recurrent activity. Whole-cell recordings were made with patch pipettes (2-4 M $\Omega$; TW150-4 glass; World Precision Instruments) filled with the following solution (in $\mathrm{mM}$ ): $130 \mathrm{Cs}^{+}$methanesulfonate, $4 \mathrm{NaCl}, 10 \mathrm{BAPTA}, 10 \mathrm{HEPES}, 4 \mathrm{MgATP}$, and 0.4 NaGTP; $290 \mathrm{mOsm}, \mathrm{pH}$ 7.4. NMDAR-mediated EPSCs were isolated by recording at $+30 \mathrm{mV}$ to relieve magnesium block and in the presence of the AMPAR/kainate receptor antagonist 2,3-dihydroxy-6nitro-7-sulfamoylbenzo[f] quinoxaline (NBQX) $(10 \mu \mathrm{M})$.

Synaptically evoked transporter currents (STCs) were recorded from CA1 astrocytes identified in stratum radiatum by their small cell bodies, low input resistance, and negative resting membrane potential (approximately -90 $\mathrm{mV}$ ). Whole-cell patch pipettes were filled with the following solution (in mM: $120 \mathrm{~K}^{+}$methanesulfonate, 10 EGTA, 20 HEPES, 2 MgATP, $0.2 \mathrm{NaGTP}$; $290 \mathrm{mOsm}, \mathrm{pH}$ 7.4). Astrocytes were held at $-90 \mathrm{mV}$. Excluding D-serine, transporter currents were recorded in the same solution (i.e., including 10 $\mu \mathrm{M}$ NBQX) as NMDAR EPSCs plus the NMDAR antagonist (RS)-3-(2carboxypiperazin-4-yl)-propyl-1-phosphonic acid (RS-CPP) $(5 \mu \mathrm{M})$ and the adneosine-1 receptor antagonist DPCPX (8-cyclopentyl-1,3-dipropylxanthine) $(1 \mu \mathrm{M})$. DL-Threo- $\beta$-benzyloxyaspartic acid (TBOA) $(50-100 \mu \mathrm{M})$, a competitive, nonsubstrate transporter antagonist, was added at the end of each experiment to block the STC and isolate the slow, potassium-mediated current (Bergles and Jahr, 1997).

EPSCs and STCs were recorded with an Axopatch 1D amplifier (Molecular Devices). Series resistance was not compensated. Synaptic events were evoked by brief electrical pulses $(50 \mu \mathrm{s} ; 20-500 \mu \mathrm{A})$ delivered at 0.1 $\mathrm{Hz}$ by a bipolar stimulating electrode (115 $\mu \mathrm{m}$ pole spacing; Frederick Haer Company) placed in the stratum radiatum, $100-200 \mu \mathrm{m}$ from the recording area. Series resistance was monitored throughout each experiment with brief $(10 \mathrm{~ms}) 1 \mathrm{mV}$ pulses that preceded synaptic stimulation. Data were acquired with a 16 bit analog-to-digital board (Instrutech) controlled by software written in IgorPro (Wavemetrics). Signals were filtered at $2 \mathrm{kHz}$ and digitized at $5 \mathrm{kHz}$.
Physiological analysis. Synaptic responses were analyzed in IgorPro. EPSCs and STCs were averaged from 10 to 20 sweeps and 5 to 10 sweeps per condition, respectively. Only neuronal recordings in which the series resistance remained stable and $\leq 20 \mathrm{M} \Omega$ were analyzed.

STCs were elicited with alternating single and paired (50 ms interval) stimuli. The response to the second stimulation in the pair was isolated by subtracting the single response from the response to paired stimulation. The single response was then subtracted from the isolated second response (Diamond, 2005), yielding the facilitated STC and eliminating most of the slowly decaying, TBOA-insensitive current thought to result from extracellular potassium accumulation (Bergles and Jahr, 1997). Any remaining slow current was eliminated by subtracting a residual current waveform from the STC. The residual waveform was approximated by fitting an exponentially rising function scaled to the STC amplitude $(A)$ at $350 \mathrm{~ms}$ after stimulation as follows:

$$
A\left(1-\exp \left(\frac{-t}{\tau_{\text {rise }}}\right)\right)
$$

$\tau_{\text {rise }}$ was determined in separate experiments by fitting STCs recorded in $100 \mu \mathrm{M}$ TBOA with an exponential functional. $\tau_{\text {rise }}$ was $13.1 \pm 9.11 \mathrm{~ms}$ $(n=5)$ in neonatal astrocytes and $4.75 \pm 2.42 \mathrm{~ms}(n=3)$ in juvenile astrocytes.

The centroid was calculated as the ratio of the first moment of the STC waveform to its area (Bracewell, 2000) as follows:

$$
\langle t\rangle=\frac{\int t f(t) d t}{\int f(t) d t}
$$

where the onset of the STC was set to $t=0$.

For deconvolution analysis, an exponentially rising and decaying function was fit to STCs to obtain smooth waveforms as follows:

$$
f(t)=A\left(1-\exp \left(-\frac{t-t_{0}}{\tau_{r}}\right)\right)^{n}\left[\exp \left(-\frac{t-t_{0}}{\tau_{f}}\right)+\exp \left(-\frac{t-t_{0}}{\tau_{s}}\right)\right] .
$$

(For a complete description of centroid and deconvolution analysis, see Diamond, 2005.)

SDS-PAGE and Western blotting. Hippocampi were dissected from anesthetized C57BL/6 mice, homogenized for $30 \mathrm{~s}$ in cold PBS, containing $1 \mathrm{~mm}$ PMSF, 1 mm EDTA, 5 mM DTT, and Complete Protease Inhibitor Cocktail Tablets ( 1 tablet/10 ml of PBS; Roche Diagnostics). Homogenates were divided into aliquots and stored at $-20^{\circ} \mathrm{C}$ or solubilized by adding $20 \%$ SDS $(2 \% \mathrm{w} / \mathrm{v}$ final concentration) followed by incubation and frequent mixing at room temperature for $20 \mathrm{~min}$. Protein concentrations were determined by BCA protein assay (Thermo Fisher Scientific).

Solubilized homogenate proteins $(25 \mu \mathrm{g})$ were loaded onto SDSPAGE gels ( $4 \sim 12 \%$ Bis-Tris gradient gels; Invitrogen), separated by electrophoresis, and transferred to nitrocellulose membranes (Invitrogen). Membranes were pretreated in TBST (TBS with $0.1 \%$ Tween 20) containing $10 \%$ nonfat milk for $1 \mathrm{~h}$ at room temperature before being probed with primary antibodies overnight at $4^{\circ} \mathrm{C}$. The following primary antibodies were diluted in TBST with $2 \%$ nonfat milk: guinea pig polyclonal anti-GLAST (1:5000; Millipore Bioscience Research Reagents), guinea pig polyclonal anti-GLT-1 (1:3000; Millipore Bioscience Research Reagents; specifically anti-GLT-1a variant) (Chen et al., 2002), and mouse monoclonal anti-actin (1:5000; Sigma-Aldrich). Membranes were washed (three times) with TBST before treatment with the following concentrations (diluted in $2 \%$ nonfat milk TBST) of species-specific, horseradish peroxidase-conjugated, secondary antibodies for $2 \mathrm{~h}$ at room temperature: goat anti-guinea pig (1:2000; Alpha Diagnostic) and sheep anti-mouse (1:2000; Millipore). Probed membranes were washed (three times) with TBST before peroxidase activity was assayed with enhanced chemiluminescence (ECL kit; Thermo Fisher Scientific).

Chemiluminescence was detected by exposing the ECL-treated blots to x-ray film. Exposed film was digitally scanned and densitom- 
etry of the images was performed using Gel-Pro Analyzer 4.5 software (Media Cybernetics). The intensity of detected transporter protein bands was normalized to the intensity of the actin bands detected in the same sample lane.

Immunohistochemistry. The same dissection procedure used for preparing slices was used to dissect hippocampi. Hippocampi were fixed in PBS containing 4\% paraformaldehyde at room temperature (RT) for $2 \mathrm{~h}$, rinsed with PBS, and cryoprotected in PBS containing 30\% sucrose overnight at $4^{\circ} \mathrm{C}$. Transverse sections ( $20 \mu \mathrm{m}$ thick) were serially cut on a cryostat and collected on glass slides. Sections were permeabilized and blocked by incubating with blocking buffer $(0.3 \%$ Triton X-100, 2\% bovine serum albumin, and $0.1 \%$ sodium azide in PBS) for $1 \mathrm{~h}$ at RT. One or more of the following primary antibodies diluted in blocking buffer were used to probe sections overnight at RT: polyclonal rabbit anti-S100 (1:500; Dako), polyclonal guinea pig anti-GLAST (1:600; Millipore Bioscience Research Reagents), or polyclonal guinea pig anti-GLT-1 (1:250; Millipore Bioscience Research Reagents). Slides were washed (three times; $5 \mathrm{~min} /$ wash) with PBS before being incubated for $2 \mathrm{~h}$ at RT with the following secondary antibodies, diluted in blocking buffer: donkey anti-rabbit Alexa Fluor 555 (1:500; Invitrogen) or donkey anti-guinea pig FITC (1:400; Jackson ImmunoResearch). After rinsing with PBS, slides were incubated with FluoroPure grade Hoechst 33258 [4',6-diamidino2-phenylindole (DAPI); Invitrogen] diluted 1:1000 in $10 \mathrm{~mm}$ bicarbonate buffer (final concentration, $>2 \mu \mathrm{g} / \mathrm{ml}$ ) for $10 \mathrm{~min}$ at RT. Slides were washed with PBS and treated with Vectashield mounting medium (Vector Laboratories) before being mounted with a glass coverslip. Immunoreactivity was visualized and acquired with a confocal laser-scanning microscope (Zeiss LSM 510; 10, 20, and $40 \times$ objectives).

Astrocytes were counted from slices triply stained with anti-S100B, anti-GLAST, and DAPI. Serial $Z$-sections ( $2 \mu \mathrm{m}$ thick) were imaged from two neighboring areas in the CA1 region of each slice with a $40 \times(1.3$ numerical aperture) oil-immersion objective. The number of sections taken in each field depended on tissue depth, which was estimated by measuring the $z$-axis borders of anti-GLAST fluorescence with the confocal pinhole size set at 1 airy unit. Projections of pseudocolored antiS100 (red) and DAPI (green) serial $Z$-section images were made in ImageJ, resulting in yellow-colored nuclei. Viewed side by side with the original serial $Z$-sections, triply colored anti-S100 (red), anti-GLAST (green), and DAPI (blue), yellow-colored nuclei were only counted if they showed anti-GLAST staining in two or more continuous sections, preferably surrounding nuclei. Counting boxes were drawn around the stratum radiatum that included at least the neighboring row of pyramidal cells. Because CA1 is smaller in neonates, $40 \times$ images of neonatal slices often captured the pyramidal cell and stratum lacunosum-moleculare layers surrounding the stratum radiatum, whereas images from juvenile slices only captured the pyramidal cells and $80 \pm 11 \%(n=27$ images $)$ of stratum radiatum. To normalize for these size differences, counting boxes were drawn around the first $80 \%$ the neonatal stratum radiatum before reaching the stratum lacunosum-moleculare. The area of juvenile counting boxes $\left(82,876 \pm 13,813 \mu \mathrm{m}^{2}\right)$ was greater $(p \leq 0.001)$ than that of neonatal boxes $\left(54,102 \pm 18,836 \mu \mathrm{m}^{2}\right)$, whereas the depths of each $40 \times$ field, estimated by summing of the $Z$-sections containing antiGLAST staining, were similar at each age $(21 \pm 4 \mu \mathrm{m}$ for neonatal slices and $24 \pm 5 \mu \mathrm{m}$ for juvenile slices). Astrocyte nuclei counts were expressed as the number of astrocytes/volume (in cubic micrometers) $\times$ 106 estimated for each counting box.

Electron microscopy. Three hours after they were prepared, slices (300 $\mu \mathrm{m}$ thick) were fixed for $1 \mathrm{~h}$ at room temperature in fixative solution $(2 \%$ glutaraldehyde and $2 \%$ paraformaldehyde in $0.1 \mathrm{~N}$ cacodylate buffer) and then stored overnight at $4^{\circ} \mathrm{C}$. Slices were washed three times with $0.1 \mathrm{~N}$ cacodylate buffer before they were trimmed to orient the middle of the CA1 region for sectioning. Samples were treated with $1 \%$ osmium tetroxide on ice for $1 \mathrm{~h}$, en bloc mordanted with $0.25 \%$ uranyl acetate overnight at $4^{\circ} \mathrm{C}$, washed, dehydrated with a graded series of ethanol washes, and embedded in epoxy resins. Thin sections $(70-90 \mathrm{~nm}$ ) were cut parallel to pyramidal cell dendrites projecting into the stratum radiatum, counterstained with lead citrate and uranyl acetate, and examined on a JEOL 1200 EX transmission electron microscope. Sampling was restricted to the middle of slices, where there was no slicing damage. Two sets of 15 consecutive images were collected from each slice at 10,000 $\times$ magnification $(4.68 \times 4.68 \mu \mathrm{m})$ with a CCD digital camera system (XR-100 from AMT). One image set was collected between 30 and $80 \mu \mathrm{m}$ away from the cell body layer, whereas the second was collected between 85 and $140 \mu \mathrm{m}$ away. Asymmetric synapses, containing presynaptic vesicles and a postsynaptic density, were counted in each image. Three images per subset were chosen randomly for extracellular space measurements (Underwood, 1970). A grid of $500 \times 500$ pixel boxes $(34 \times 34 \mathrm{~mm})$ was laid over a $2500 \times 2500$ pixel $(170 \times 170 \mathrm{~mm})$ portion of each image. The extracellular area of 10 randomly chosen grid boxes was traced by hand and measured in ImageJ. Summation of these measurements provided the extracellular volume fraction of each image. Average extracellular volume fractions were obtained by pooling 18 volume fraction estimates from 3 animals (6 estimates/animal).

Diffusion simulations. Transmitter diffusion and uptake was modeled in Matlab (The MathWorks) as described previously (Diamond, 2005). During a simulated synaptic event, 2000 glutamate molecules were released from within a 320-nm-diameter (20-nm-thick) synaptic cleft and moved independently through space on a random walk ( $1 \mu$ s time step). The diffusion coefficient $D, 0.76 \mu \mathrm{m}^{2} \mathrm{~ms}^{-1}$ (in aqueous solution at $25^{\circ} \mathrm{C}$ ) (Longsworth, 1953), was reduced by two-thirds to account for diffusion in extracellular fluid (Nielsen et al., 2004) and increased 1.3fold to account for the $35^{\circ} \mathrm{C}$ recording temperature (Hille, 1984). Diffusion was two-dimensional within the synaptic cleft (Barbour and Häusser, 1997) and three-dimensional through the extrasynaptic space, which was modeled as an isotropic medium [extracellular volume fraction, 0.18 or 0.377 ; tortuosity $(\lambda), 1.55$ ] (Rusakov and Kullmann, 1998).

Glutamate transporters were simulated with a simplified Markov representation of EAAT2 (Bergles et al., 2002) $\left(V_{\mathrm{m}}=-95 \mathrm{mV}\right.$ ) (Diamond, 2005). Transition rates derived from room temperature experiments (Bergles et al., 2002) were multiplied by 3 to approximate kinetics at $35^{\circ} \mathrm{C}$. NMDAR activation (Lester and Jahr, 1992) was simulated in ChanneLab (Synaptosoft) using the glutamate concentration waveforms generated by diffusion simulations. Transition rates in the NMDAR model were increased by a factor of 3 to simulate $35^{\circ} \mathrm{C}$; the maximal $P_{\mathrm{o}}$ for the simulated NMDARs was 0.29 .

Statistics. Except where noted, all data are reported as averages \pm SDs. Lowercase $p$ values represent paired $t$ tests used to compare averages within groups, whereas uppercase $P$ values represent unpaired $t$ tests used to compare averages between neonatal and juvenile samples.

\section{Results}

\section{Developmental changes in glial transporter expression and neuropil ultrastructure}

Previous reports have shown that developmental increases in hippocampal GLAST and GLT-1 protein levels coincide with changes in uptake capacity and clearance of synaptically released glutamate in the rat (Schmidt and Wolf, 1988; Bergles and Jahr, 1997; Furuta et al., 1997; Ullensvang et al., 1997; Kugler and Schleyer, 2004; Diamond, 2005). We found that mouse hippocampal GLAST and GLT-1 protein levels also increase rapidly during early postnatal development (Fig. $1 A-C$ ), as suggested by gene expression studies (Shibata et al., 1996; Sutherland et al., 1996; Regan et al., 2007). Western blots of hippocampal homogenates from P4, P5, and P6 mice indicated that GLAST and GLT-1 protein levels were lower than levels detected in juvenile tissue $(\sim 23 \pm 7 \%$ of P15 GLAST levels and $\sim 12 \pm 3 \%$ of P15 GLT-1) (Fig. 1A). Immunofluorescence indicated that these increases were evenly distributed throughout hippocampal slices (Fig. $1 \mathrm{~B}$ ). Astrocyte densities were similar in the CA1 regions of neonatal and juvenile slices (neonate, $23 \pm 7$ astrocytes $/ \mathrm{mm}^{3}$; juvenile, $26 \pm 7$ astrocytes $/ \mathrm{mm}^{3} ; P=0.052$ ) (Fig. $1 C$ ), consistent with previous work (Ogata and Kosaka, 2002) (Fig. 1C). These results suggest that astrocyte uptake capacity increases in mice during early development, potentially speeding the clearance of synaptically released glutamate. 
A
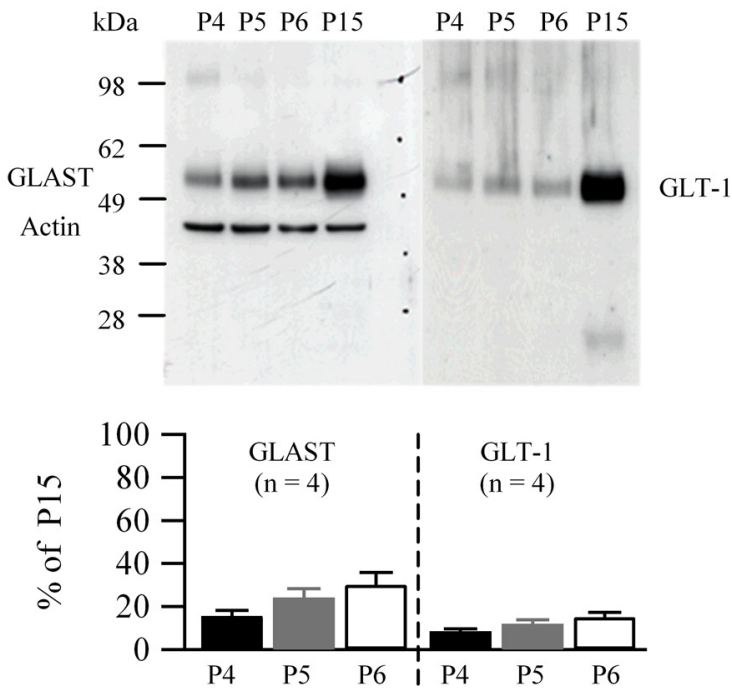

B
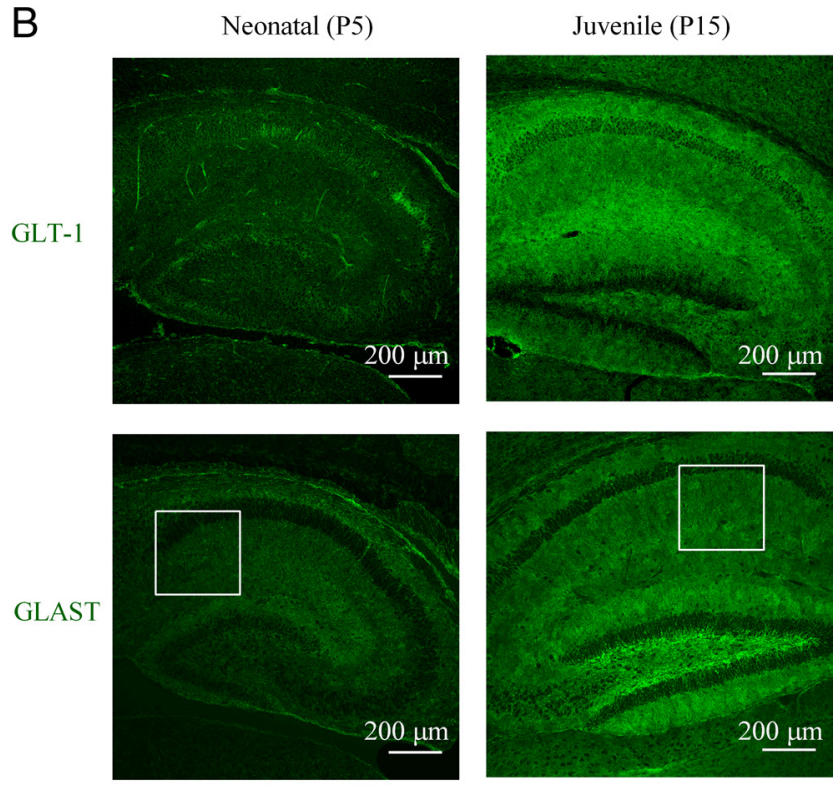

C
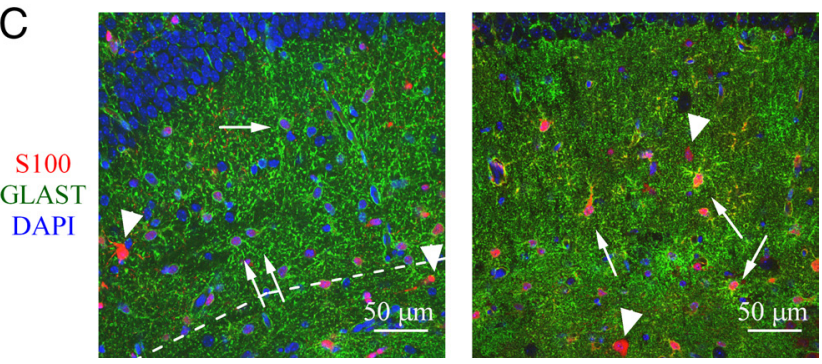

Figure 1. Glial glutamate transporter expression increases during early postnatal development. $\boldsymbol{A}$, Western blots of GLAST and GLT-1 in hippocampal protein samples from P4, P5, P6, and P15 mice. Blots were probed with anti-GLAST and anti-actin antibodies (left) and with antiGLT-1 antibody (right). Below are average GLAST and GLT-1 protein levels from P4, P5, and P6 mice (4 mice/age), measured by chemiluminescence and expressed as a percentage of P15 levels. Error bars indicate SEM. $\boldsymbol{B}$, Immunostaining for the glial transporter proteins, GLAST and GLT-1. Hippocampal slices from a neonatal (P5, left panels) and a juvenile mouse (P15, right panels) were stained with anti-GLT-1 (top panels) or anti-GLAST (bottom panels) antibodies. Images were taken with a $10 \times$ objective. The boxes represent $40 \times$ images shown below. $\boldsymbol{C}$, Immunostaining and counting of CA1 astrocyte nuclei. Anti-GLAST-stained slices were also stained with antibodies against the glial marker $\$ 100$ and with the nuclear stain DAPI. Shown are examples of individual $z$-section images ( $2 \mu \mathrm{m}$ thick) taken from the boxes shown in $\boldsymbol{B}$. The arrows point to examples of astrocyte nuclei, triply labeled with anti-S100 (red) and anti-GLAST (green) antibodies and DAPI (blue), whereas arrowheads point to examples of anti-S100 and
Structural parameters like extracellular volume fraction or synaptic density can also influence the glutamate concentration time course, ultimately leading to measurable effects on synaptic transmission (Iino et al., 2001; Piet et al., 2004; Cathala et al., 2005). Electron micrographs of CA1 stratum radiatum obtained from acute hippocampal slices indicated that stratum radiatum ultrastructure changes greatly during synaptic maturation (Fig. 2 ). There was a fivefold increase in the density of asymmetric synapses (P5, $0.08 \pm 0.08$ synapses $/ \mu \mathrm{m}^{2} ; \mathrm{P} 14,0.41 \pm 0.16$ synapses $\left./ \mu \mathrm{m}^{2} ; P<0.001\right)$ and an approximately twofold decrease in extracellular volume fraction $(\alpha ; \mathrm{P} 5,0.38 \pm 0.09 ; \mathrm{P} 14,0.18 \pm$ $0.05 ; P<0.001)$. We would expect any change in synaptic density caused by the slicing procedure (Fiala et al., 2003) to affect both ages similarly (Kirov et al., 2004). Previous studies reported similar ultrastructural trends in hippocampi from perfused rats (Steward and Falk, 1991; Boyer et al., 1998; Fiala et al., 1998), suggesting that our results reflect common developmental changes in rodent brains.

\section{Glutamate uptake gets faster during the second postnatal week}

To investigate whether the developmental changes reported in Figures 1 and 2 influence uptake of synaptically released glutamate, we recorded STCs from astrocytes in acute hippocampal slices from neonatal (P4-P6) and juvenile (P13-P15) mice. Responses were evoked by single and paired ( $50 \mathrm{~ms}$ interval) Shaffer collateral stimulation in the presence of AMPAR, GABAR, and NMDAR antagonists, producing inward currents with fast and slow components (Fig. 3A). The fast component of neonatal and juvenile responses was blocked completely by the competitive glutamate transporter antagonist TBOA (50 or $100 \mu \mathrm{M})$, indicating transporter-mediated glutamate uptake, whereas the small, slower component was insensitive to TBOA, as previously reported (Bergles and Jahr, 1997; Diamond, 2005) (Fig. 3A). These results indicated that uptake of synaptically released glutamate occurs at both ages despite relatively low transporter expression in neonates (Bergles and Jahr, 1997). At both ages, paired stimulation evoked a larger TBOA-sensitive current on the second pulse, indicating that more glutamate was synaptically released in response to the second pulse than to the first (Bergles and Jahr, 1997). Paired-pulse facilitation was greater in juvenile slices $(265 \pm 25 \%$ of first STC; $n=11)$ than in neonatal slices $(223 \pm 16 \%$ of first STC; $n=12 ; p=0.0002)$, suggesting that release probability decreases during synaptic maturation, as reported previously in rat (Muller et al., 1989; Bolshakov and Siegelbaum, 1995; Wasling et al., 2004) (but see Hsia et al., 1998).

The STC time course provides a relative indication of how rapidly synaptically released glutamate is taken up from the extracellular space (Diamond, 2005). We compared STCs in neonatal and juvenile slices after minimizing contamination by the slow, TBOA-insensitive component (Diamond, 2005) (see Materials and Methods). The $20-80 \%$ rise time, exponential decay time constant, and centroid ( $\langle t\rangle$; the temporal "center of mass" reflecting both the rise and decay) of STCs were faster in juvenile slices than in neonatal slices (Fig. $3 C, D$ ), suggesting that uptake capacity increases with age.

\section{$\leftarrow$}

DAPI-labeled cells with no anti-GLAST staining. Only the triply labeled nuclei were counted. Note the ring of anti-GLAST staining that was a hallmark of triply labeled astrocyte nuclei at both ages. Counting in the neonatal slice was restricted to the area between the pyramidal cell layer and the dashed white line. 

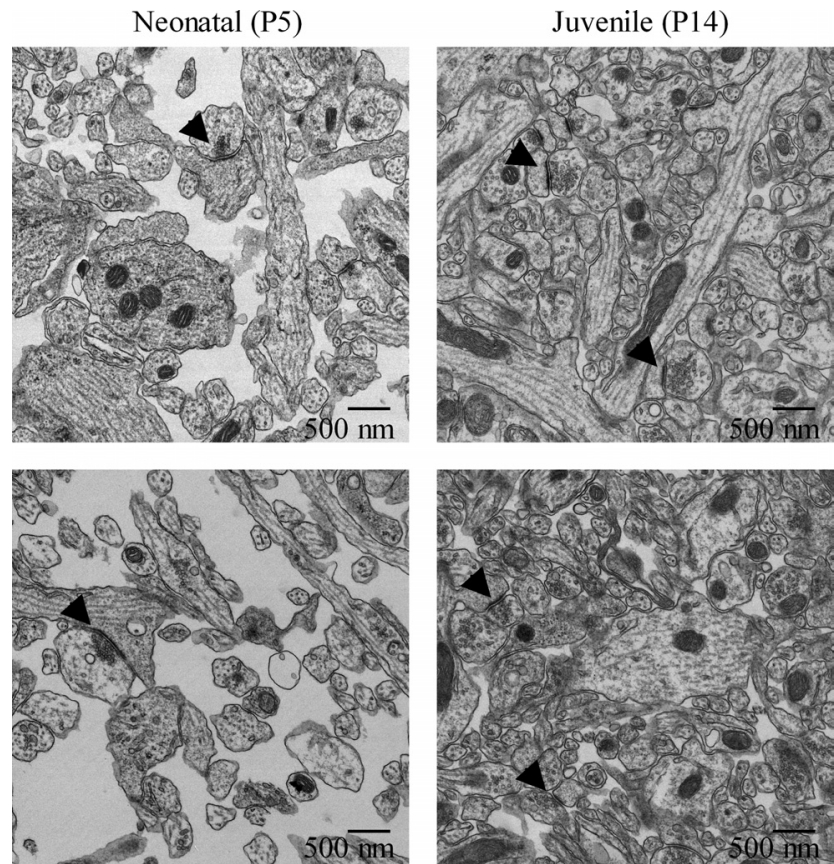

Figure 2. The hippocampus undergoes ultrastructural changes during early postnatal development. Electron micrographs of hippocampal slices from neonatal (left) and juvenile (right) mice. The arrowheads point to examples of asymmetric synapses. Calculated extracellular volume fractions for the neonatal sections shown are 0.31 (top) and 0.43 (bottom) and for the juvenile sections are 0.15 (top) and 0.21 (bottom).

Previous studies reported that GLT-1 expression increases more than GLAST expression during the second postnatal week (Furuta et al., 1997; Kugler and Schleyer, 2004). We tested whether differential expression of GLAST and GLT-1 influenced changes in STC time course during this period by recording STCs in the presence of dihydrokainate (DHK), a GLT-1-selective competitive antagonist. DHK reduced the amplitudes and slowed the decays of STCs to the same extent in neonatal ( $57 \pm 10 \%$ control peak; $194 \pm 22 \% \operatorname{control}\langle t ; ; n=6$ ) and in juvenile (58 \pm $4 \%$ control peak; $191 \pm 13 \%$ control $\langle t\rangle ; n=6$ ) slices (Fig. $3 E$ ) (neonate vs juvenile, $P=0.73$ ), suggesting that GLT-1 contributes equally to uptake throughout synaptic maturation and that any intrinsic kinetic differences between GLAST and GLT-1 remain the same (Arriza et al., 1994; Wadiche et al., 2006).

STCs do not reflect the exact time course of glutamate uptake, because they are slowed by asynchronous transmitter release and electronic filtering (Diamond, 2005; Scimemi et al., 2009). To determine quantitatively how uptake capacity changes during development, it becomes necessary to account for these distortions when analyzing the time course of the STC. The distortions behave, to a first approximation, as a linear filter that can be characterized and deconvolved from the STC wave form, yielding a more accurate estimation of the time course of uptake (Diamond, 2005). A related approach exploits the fact that centroids add under convolution (Bracewell, 2000), in other words, as follows:

$$
\langle t\rangle_{\mathrm{STC}}=\langle t\rangle_{\text {uptake }}+\langle t\rangle_{\text {filter }} \text {. }
$$

This equation shows that, although increased transporter concentration results in more rapid uptake, the STC can never be faster than the filter (see a hypothetical example in Fig. 4A). If, as in a linear system, the rate of uptake is proportional to transporter concentration, plotting $\langle t\rangle_{\text {STC }}$ versus the inverse of the transporter concentration ([transporter $]^{-1}$ ) yields a linear rela-
A

Neonatal STCs

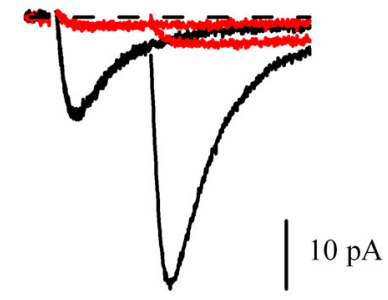

B

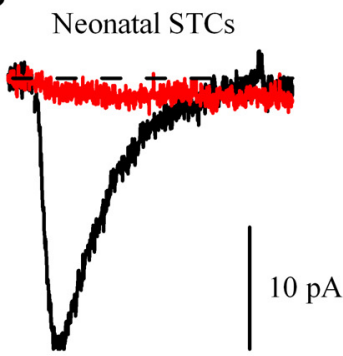

C

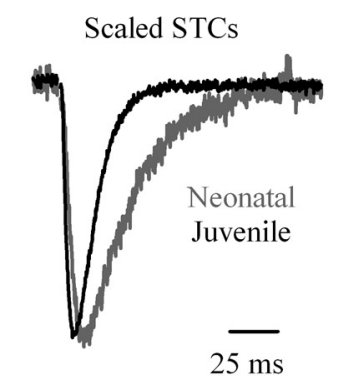

$\mathrm{D}$

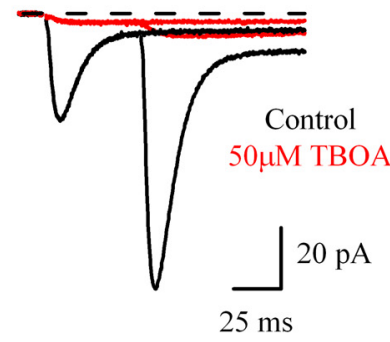

Juvenile STCs

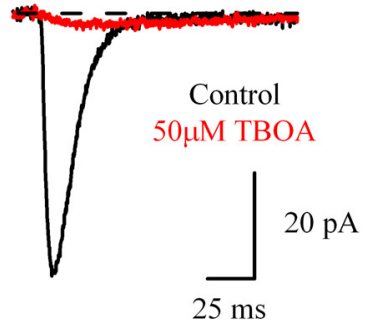

$\mathrm{D}$

Neonatal $(\mathrm{n}=11)$

Juvenile $(\mathrm{n}=15)$

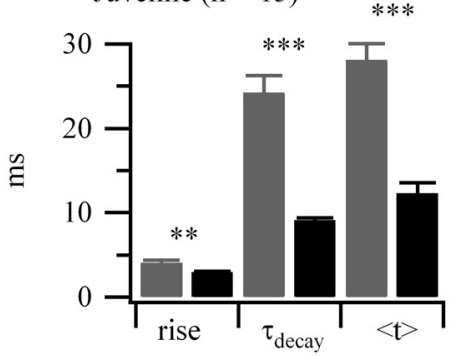

$E$
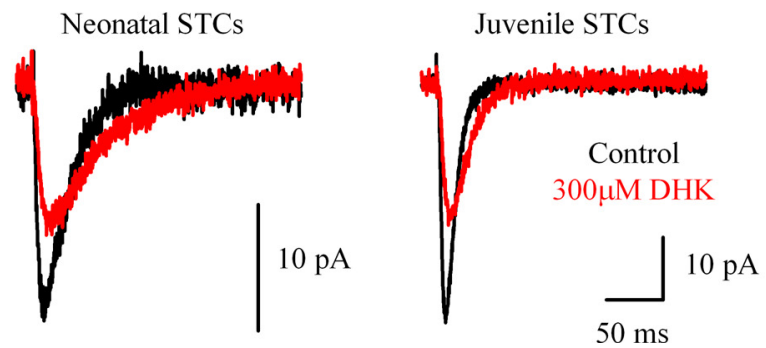

Figure 3. STCs speed up during early postnatal development. A, Average synaptic responses recorded in astrocytes from a neonatal and a juvenile hippocampal slice. Whole-cell voltageclamp currents recorded from stratum radiatum astrocytes in response to single and paired Schaffer collateral stimulation evoked in control solution and in TBOA $(50 \mu \mathrm{m})$. B, Average facilitated transporter currents derived from the recordings shown in $A$. $C$, Neonatal and juvenile control STCs shown in $\boldsymbol{B}$ were normalized to their peak amplitude and overlaid to compare their shapes. $\boldsymbol{D}$, Average rise $(20-80 \%)$, decay $\left(\tau_{\text {decay }}\right)$, and centroid $(\langle t\rangle)$ of STCs recorded in neonatal and juvenile slices. Unpaired $t$ tests were used to compare differences between neonatal and juvenile measurements ( $\left.{ }^{* *} P=0.004 ; * * P \leq 0.001\right)$. Error bars indicate SEM. E, Average STCs recorded in astrocytes from a neonatal (left) and juvenile (right) hippocampal slice in control solution (black traces) and in the presence of $300 \mu \mathrm{m}$ DHK (red traces).

tionship (Fig. 4B) (Diamond, 2005) that intercepts the $y$-axis at $\left\langle t_{\text {filter }}\right.$ (i.e., $\langle t\rangle_{\text {STC }}$ when uptake is infinitely fast) and has a slope equal to $\langle t\rangle_{\text {uptake }}$ (i.e., shallower slope $=$ faster uptake) (Fig. $4 B$ ).

We performed this centroid analysis on STCs recorded in control conditions and after partially blocking uptake with $10 \mu \mathrm{M}$ TBOA in neonatal and juvenile slices (Fig. 4C,D). TBOA reduced the functional transporter concentration to an extent that was estimated 

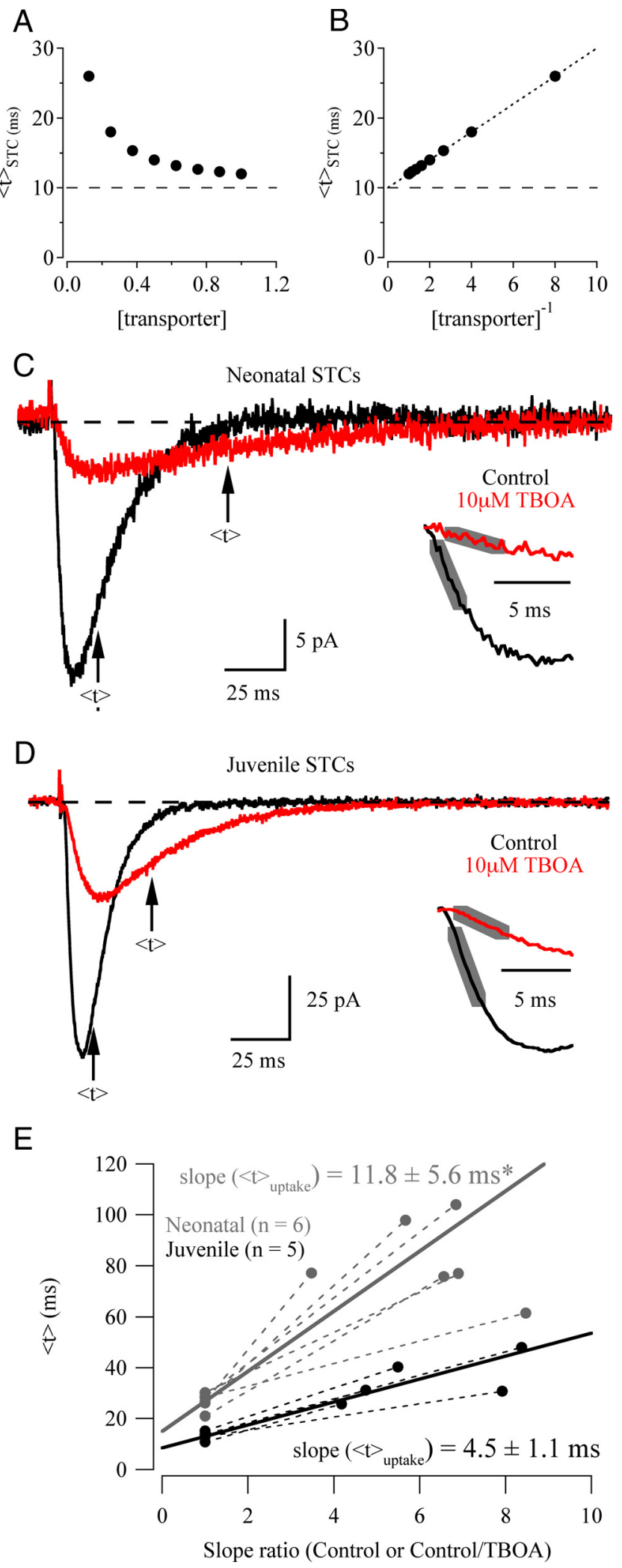

Figure 4. Clearance of synaptically released glutamate becomes faster during early postnatal development. $\boldsymbol{A}, \boldsymbol{B}$, Hypothetical plots of STC centroid versus transporter concentration ([transporter]) $(\boldsymbol{A})$ or of the inverse of transporter concentration ([transporter] $\left.{ }^{-1}\right)(\boldsymbol{B})$. The dashed lines represent the centroid of the filter. The dotted line in $\boldsymbol{B}$ is a linear regression to the data. $\boldsymbol{C}, \boldsymbol{D}$, Average STCs recorded in astrocytes from neonatal $(\boldsymbol{C})$ or juvenile $(\boldsymbol{D})$ slices in control solution (black traces) or in the presence of $10 \mu \mathrm{m}$ TBOA (red traces). The arrows indicate the centroid $(\langle t\rangle)$ locations of each response. The insets show the first $10 \mathrm{~ms}$ of the STCs. The thick gray bars represent the initial slope measured for each STC. $\boldsymbol{E}$, Estimation of glutamate clearance from the decrease in the STC initial slope (Fig. 4C,D, inset), which is proportional to uptake capacity (Diamond, 2005). As predicted, reducing uptake also slowed the STC (i.e., increased $\left\langle t_{\text {STC }}\right.$ ). Plotting $\left\langle t_{\text {STC }} \text { versus [transporter }\right]^{-1}$ indicated that glutamate clearance was $\sim 2.5$ times faster in juvenile slices than in neonatal slices $(P=0.02)$ (Fig. $4 C$ ). These values were confirmed by deconvolution-based analysis of the same data (Diamond, 2005) (juvenile clearance, $4.4 \pm$ $1.2 \mathrm{~ms}, n=5$; neonatal clearance, $15.4 \pm 8.4 \mathrm{~ms}, n=4 ; P=0.01$ ), and similar results were obtained when STCs were reduced by 300 $\mu \mathrm{M}$ DHK (data not shown).

\section{Developmental changes in extracellular volume fraction influence the glutamate concentration time course}

The results presented thus far indicate that STCs get faster as transporter expression increases (Figs. 1, 3, 4), suggesting that developmental changes in STC waveforms reflect increased uptake capacity (Diamond, 2005). Next, we used a Monte Carlo diffusion model to examine how the extracellular volume fraction, which also changes significantly in the second postnatal week (Fig. 2), ought to affect the extent of glutamate diffusion and the time course of uptake (Fig. 5). To simulate single synaptic events, 2000 glutamate molecules were released at the center of a synaptic cleft and allowed to diffuse randomly through space until they were bound and taken up by extrasynaptic glutamate transporters (see Materials and Methods) (Diamond, 2005). Neonatal and juvenile cases were simulated using experimentally determined extracellular volume fractions (Fig. 2) and adjusting the transporter density until the time course of the simulated STC approximated the clearance time course derived from centroid analysis (Fig. 4). STCs were reproduced using an effective transporter density (i.e., accounting for the extracellular volume fraction) of $8 \mu \mathrm{M}$ for neonate and $39 \mu \mathrm{M}$ for juvenile (Fig. 5A). Considering the approximately fivefold increase in uptake capacity between juvenile and adult (Diamond, 2005), these numbers are relatively consistent with the effective concentrations derived biochemically in adult hippocampus (140-250 $\mu \mathrm{M}$ ) (Lehre and Danbolt, 1998). Decreasing the extracellular volume fraction $(\alpha)$ to the juvenile value accelerated simulated neonatal STCs (Fig. $5 A$ ), suggesting that neuropil structure also contributes to the time course of glutamate uptake. To examine this further, we repeated our centroid analysis on STCs simulated with neonatal and juvenile extracellular volume fractions (Fig. 5B), varying transporter density over the same eightfold range in both cases. The slope of the relationship, reflecting $\langle t\rangle_{\text {clearance, }}$, was larger (slower) in simulated neonatal STCs, suggesting both extracellular volume fraction and uptake capacity contribute to clearance. The reduced dilution in the juvenile neuropil accelerates binding and uptake by transporters, ultimately decreasing the average distance that glutamate diffuses before being taken up (Fig. 5C).

Despite faster uptake in the juvenile simulations, dilution was slower, leading to higher extrasynaptic glutamate levels within 1 $\mu \mathrm{m}$ of the release site (Fig. 5D). The glutamate concentration profile in the "hybrid" case (neonatal transporter levels, juvenile volume fraction) was the same as the juvenile within $500 \mathrm{~nm}$ of an

$\leftarrow$

times in neonatal and juvenile slices. Centroids measured from control and TBOA STCs are plotted as a function of STC initial slopes. The solid lines represent average slopes and $y$-intercepts of the fits from the individual cells. The slopes of the lines provide estimates of glutamate clearance times $\left(\langle t\rangle_{\text {uptake }}\right)$, which were significantly different between ages $\left({ }^{*} P=\right.$ 0.02 , unpaired $t$ test). The $y$-intercepts provide estimates of the STC filters ( $\langle t\rangle_{\text {filter }}$; neonatal filter, $15.1 \pm 6.9 \mathrm{~ms}$; juvenile filter, $8.6 \pm 1.6 \mathrm{~ms})$, which were not significantly different between ages $(P=0.07)$. 

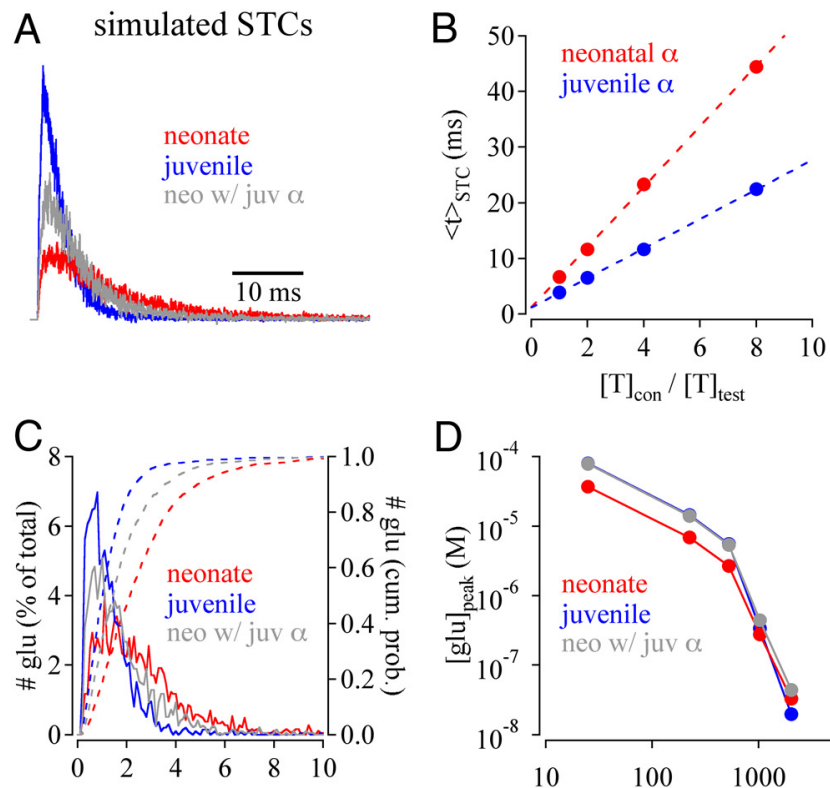

Dist. from release site $(\mu \mathrm{m})$
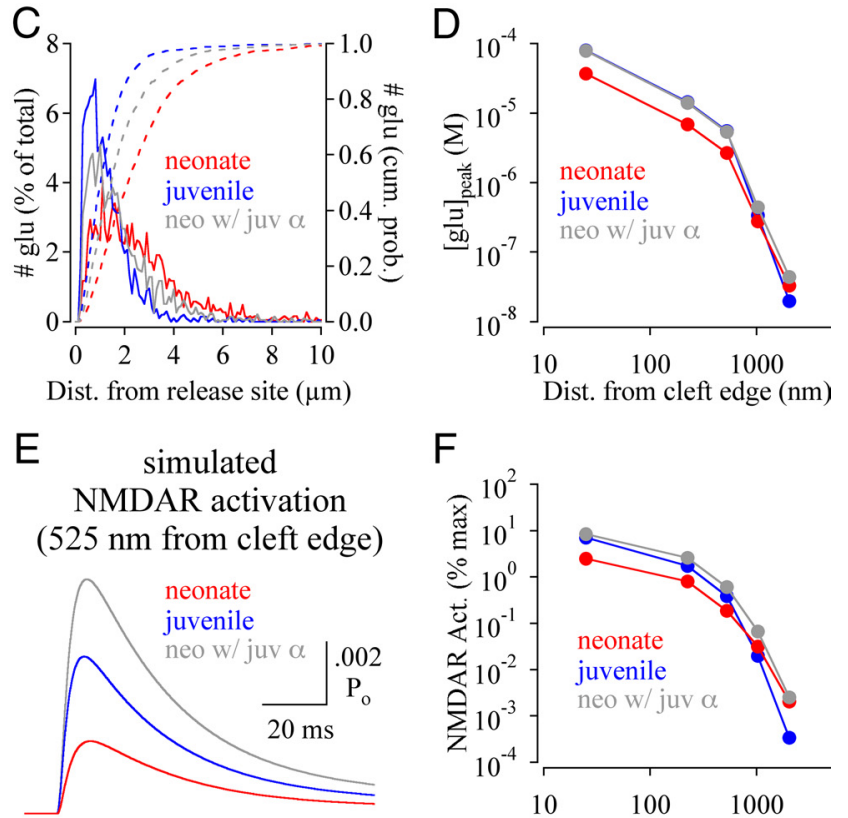

$\mathrm{F}$

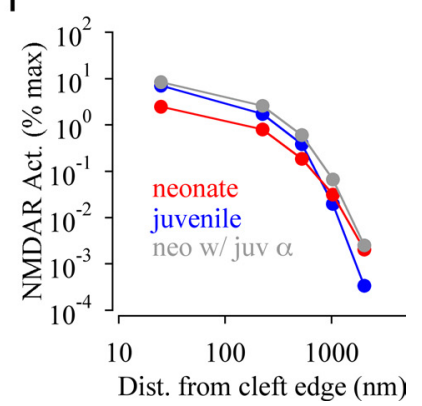

Figure 5. Simulations suggest that developmental changes in uptake capacity and extracellular volume fraction alter clearance and extrasynaptic NMDA receptor activation. $A$, Simulated STCs produced with neonatal extracellularvolume fraction ( $\alpha$ ) and lower transporter density (red traces), with juvenile $\alpha$ and higher transporter density (blue traces), and with juvenile $\alpha$ and lower (neonatal) transporter density (gray traces). $\boldsymbol{B}$, Centroid analysis of simulated STCs using neonatal and juvenile $\alpha$ values at various transporter concentrations. $\boldsymbol{C}, \boldsymbol{D}$, The conditions described in $\boldsymbol{A}$ were used in Monte Carlo simulations to predict the distance synaptically released glutamate diffuses before being transported $(\boldsymbol{C})$ and peak glutamate concentrations as a function of distance away from the cleft edge (D). The dashed lines ( $\boldsymbol{C}$ represent cumulative probability plots (right axis). $\boldsymbol{E}, \boldsymbol{F}$, The glutamate concentration predictions shown in $\boldsymbol{D}$ were used in a kinetic model of NMDARs to simulate extrasynaptic NMDAR activity $525 \mathrm{~nm}$ from the synaptic cleft edge and to predict the relative number of extrasynaptic NMDARs activated by synaptically released glutamate at various distances from the cleft edge.

active synapse, suggesting that differences in glutamate concentration in the perisynaptic region depend more on the extent of dilution in the extracellular space than on uptake (Fig. 5D). Although simulated activation of extrasynaptic NMDARs was low in both cases (Barbour, 2001), it was greater in the juvenile (Fig. $5 E, F)$, suggesting that developmental decreases in extracellular space may render NMDAR activation in juvenile slices more sensitive to changes in uptake capacity than in neonatal slices.

\section{Uptake and synaptic transmission during early postnatal development}

To test the predictions of the model experimentally, we examined the effects of transporter blockade on NMDAR EPSCs recorded from CA1 pyramidal cell neurons (Fig. 6). Recordings were made in the presence of the $\mathrm{GABA}_{\mathrm{A}} \mathrm{R}$ and AMPAR antagonists, picrotoxin $(100 \mu \mathrm{M})$ and NBQX $(10 \mu \mathrm{M})$, to isolate NMDAR EPSCs and at positive potentials $\left(V_{\mathrm{m}}=+30 \mathrm{mV}\right)$ to relieve voltage-
A

Average Responses

Neonatal Juvenile
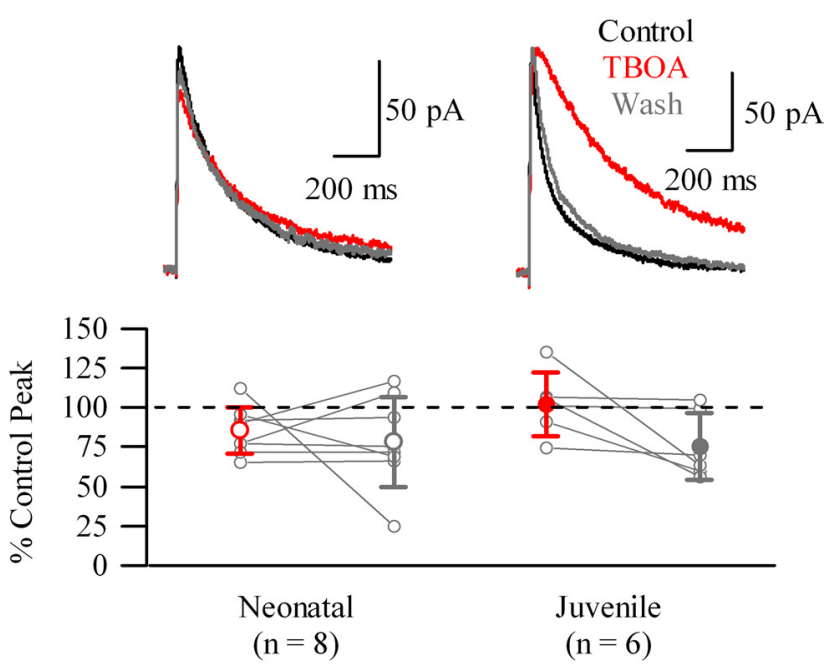

B

Normalized Responses

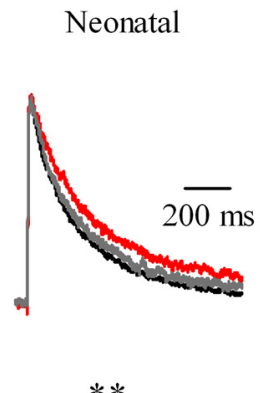

Juvenile

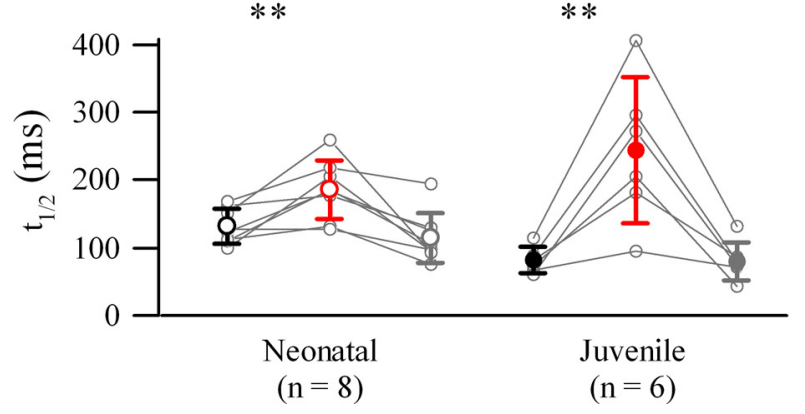

Figure 6. Glutamate uptake influences postsynaptic responses more in juvenile slices. A, NMDAR EPSCs recorded from CA1 pyramidal cell neurons in neonatal (left) and juvenile (right) slices when uptake was intact (control or wash) or completely blocked by $50 \mu \mathrm{M} \mathrm{TBOA} \mathrm{(red).} \mathrm{Below} \mathrm{is} \mathrm{a} \mathrm{graph} \mathrm{of}$ individual (thin circles) and average (thick circles and SD bars) EPS ( peak amplitudes recorded in TBOA (red) and during washout (gray) expressed as percentages of control. $\boldsymbol{B}$, The responses shown in $\boldsymbol{A}$ were normalized to their peak amplitudes to demonstrate how blocking uptake influenced EPSC decay times. Below is a graph of the individual (thin circles) and average (thick circles and SD bars) decay times $\left(t_{1 / 2}\right)$ recorded in control (black), TBOA (red), and during washout (gray). Paired $t$ tests were used to compare control and TBOA decay times $\left.{ }^{* *} p<0.001\right)$.

dependent magnesium block of NMDARs and to inactivate neuronal glutamate transporters in the recorded cell (Diamond, 2001). Stimulation of Schaffer collateral fibers elicited outward EPSCs (Fig. 6A) that were sensitive to the NMDAR antagonist $R S$-CPP (data not shown). NMDAR EPSCs decayed more rapidly in juvenile slices $\left(t_{1 / 2}=81 \pm 19 \mathrm{~ms} ; n=6\right)$ than in neonatal slices $\left(t_{1 / 2}=132 \pm 26 \mathrm{~ms} ; n=8 ; P=0.003\right)$, consistent with previous reports (Fig. 6B) (Hestrin, 1992; Kirson and Yaari, 1996). TBOA $(50 \mu \mathrm{M})$ had no effect on EPSC amplitudes recorded within the first 5 
min of application (Fig. 6A), even though glial recordings confirmed that $50 \mu \mathrm{M}$ TBOA blocks uptake within $2 \mathrm{~min}$ (time to block STCs by $>85 \%, 1.8 \pm 0.4 \mathrm{~min}$; $n=7$ ) (data not shown). During this 5 min period, TBOA moderately increased neonatal decay times $\left(t_{1 / 2}=144 \pm 37 \%\right.$ of control; $P<0.001$ ), whereas it nearly tripled juvenile decay times $\left(t_{1 / 2}=297 \pm\right.$ $108 \%$ control; $P<0.001$; neonate vs juvenile, $P=0.003$ ) (Fig. $6 B$ ), indicating that uptake regulates NMDAR activation more in the juvenile than in the neonate. Analysis was limited to the first $5 \mathrm{~min}$ of TBOA application, because EPSC amplitudes decreased during longer applications (data not shown). The mGluR (metabotropic glutamate receptor) antagonist LY341495 (2-[(1S,2S)-2-carboxycyclopropyl]-3-(9H-xanthen-9-yl)-D-alanine) $(50 \mu \mathrm{M})$ did not prevent the decline in EPSC amplitude or influence the effect of TBOA on EPSC decay times (data not shown), suggesting that presynaptic activity remained unchanged and that the decline in EPSC amplitude was likely attributable to enhanced background NMDAR activation caused by a gradual increase in ambient glutamate levels. This effect was not observed with lower TBOA concentrations $(\leq 30$ $\mu \mathrm{M})$. With uptake completely blocked by 50 $\mu \mathrm{M}$ TBOA, clearance presumably is mediated entirely by diffusion and slowed EPSCs likely reflect recruitment of distant receptors. The greater effect of TBOA on juvenile EPSC decay times is consistent with the prediction by the model that extrasynaptic NMDAR activation in juvenile slices is regulated more by glutamate uptake.

A previous report showed that blocking uptake in adult hippocampal slices prolonged NMDAR EPSC decays only in response to stronger stimulation intensities (Arnth-Jensen et al., 2002), suggesting that extrasynaptic NMDARs are more susceptible to glutamate pooling and spillover when more synapses are activated. To test whether this is also true earlier in development, we evoked NMDAR EPSCs in neonatal and juvenile slices with alternating weak and strong stimuli in control solution and in the presence of TBOA (10 $\mu \mathrm{M})$. Stimulus intensities were adjusted to evoke similar EPSC amplitudes (small, $\sim 100 \mathrm{pA}$; large, $\sim 200 \mathrm{pA}$ ) in an effort to activate comparable numbers of synapses (Fig. 7A). With uptake intact, increasing stimulus strength slowed EPSC decay times equally at both ages (neonatal strong $t_{1 / 2} /$ weak $t_{1 / 2}=1.4 \pm 0.2, n=8$; juvenile strong $t_{1 / 2} /$ weak $t_{1 / 2}=1.5 \pm 0.3, n=8$; neonatal vs juvenile, $P=0.4$ ) (Fig. $\left.7 B\right)$. At both ages, TBOA prolonged EPSC decays only in response to strong stimulation, suggesting that uptake limits extrasynaptic

B

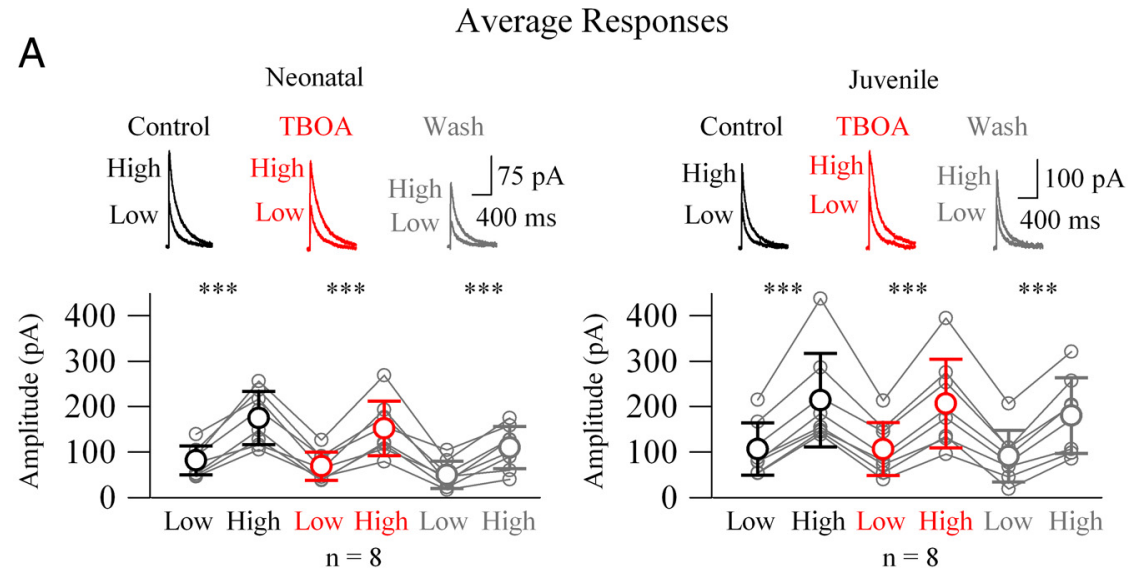

Normalized Responses
Neonatal

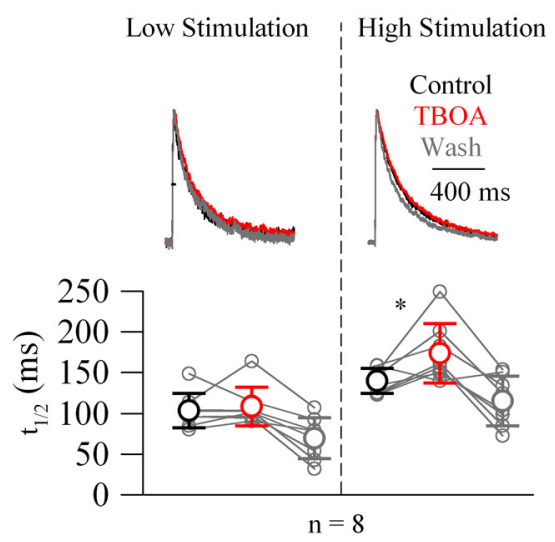

C

Regulation of EPSC Decay by Uptake

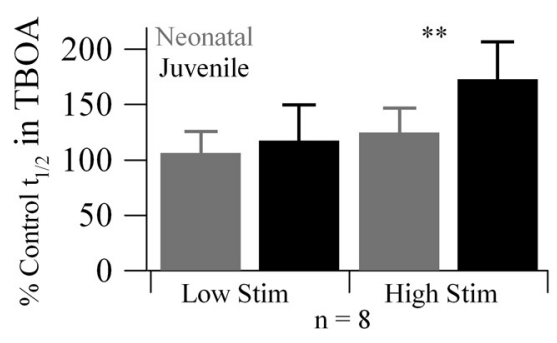

Figure 7. Uptake prevents pooling of synaptically released glutamate more in juvenile slices. $\boldsymbol{A}$, NMDAR EPSCs evoked in response to alternating high and low stimulation intensities from neonatal (left side) and juvenile (right side) CA1 pyramidal cells. EPSCs were recorded in control solution (black traces), in the presence of $10 \mu \mathrm{m}$ TBOA (red traces), and during washout (gray traces). Graphs (below) of individual (thin gray circles) and average (thick circles and SD bars) response amplitudes showing that neonatal and juvenile responses were comparable between ages. Paired $t$ tests were used to compare low- and high-response amplitudes recorded in each solution ( ${ }^{* * *} p<0.001$ ). $\boldsymbol{B}$, The EPSCs shown in $\boldsymbol{A}$ were normalized to their peak amplitudes and overlaid with the other low (left-hand groups) and high (right-hand groups) responses from each cell to compare the relative effects of blocking uptake. Graphs (below) of individual (thin gray circles) and average (think circles and SD bars) decay times from low (left side of graphs) and high (right side of responses) stimulus responses. Paired $t$ tests were used to compare control and TBOA results ( $\left.{ }^{*} p=0.02 ;{ }^{* * *} p=0.0004\right)$. $C$, Comparison of relative decay time changes caused by TBOA for low and high stimulus responses from neonatal and juvenile slices. TBOA $t_{1 / 2}$ values are expressed as percentage of control $t_{1 / 2}$. Unpaired $t$ tests determined there was a difference between neonatal and juvenile high stimulus responses ( $\left.{ }^{* *} P=0.005\right)$. $\boldsymbol{D}$, Comparison of decay time changes caused first by the NR2B-selective antagonist, ifenprodil ( $5 \mu \mathrm{m}$; left side), and then by TBOA in the presence of ifenprodil (right side). $t_{1 / 2}$ values are expressed as percentage of control $t_{1 / 2}$. Unpaired $t$ tests determined there was a difference between neonatal and juvenile responses in both conditions ( $\left.{ }^{* *} P=0.0002 ;{ }^{*} P=0.02\right)$. Error bars indicate SD.

NMDAR activation throughout early postnatal development (Fig. $7 B$ ), as previously reported in adult slices (Arnth-Jensen et al., 2002). Consistent with our previous results (Fig. 6), relative increases in $t_{1 / 2}$ (i.e., $\mathrm{TBOA} /$ control, strong stimulation) were 


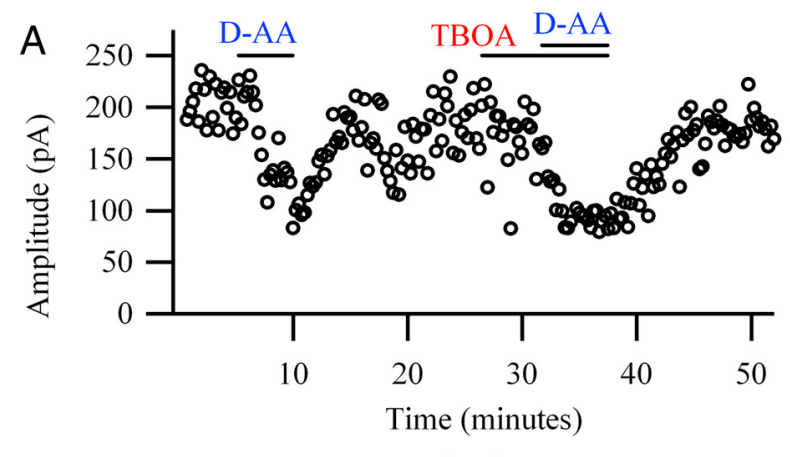

B

Normalized EPSCs Neonatal

Juvenile

Control, D-AA, TBOA, TBOA+D-AA
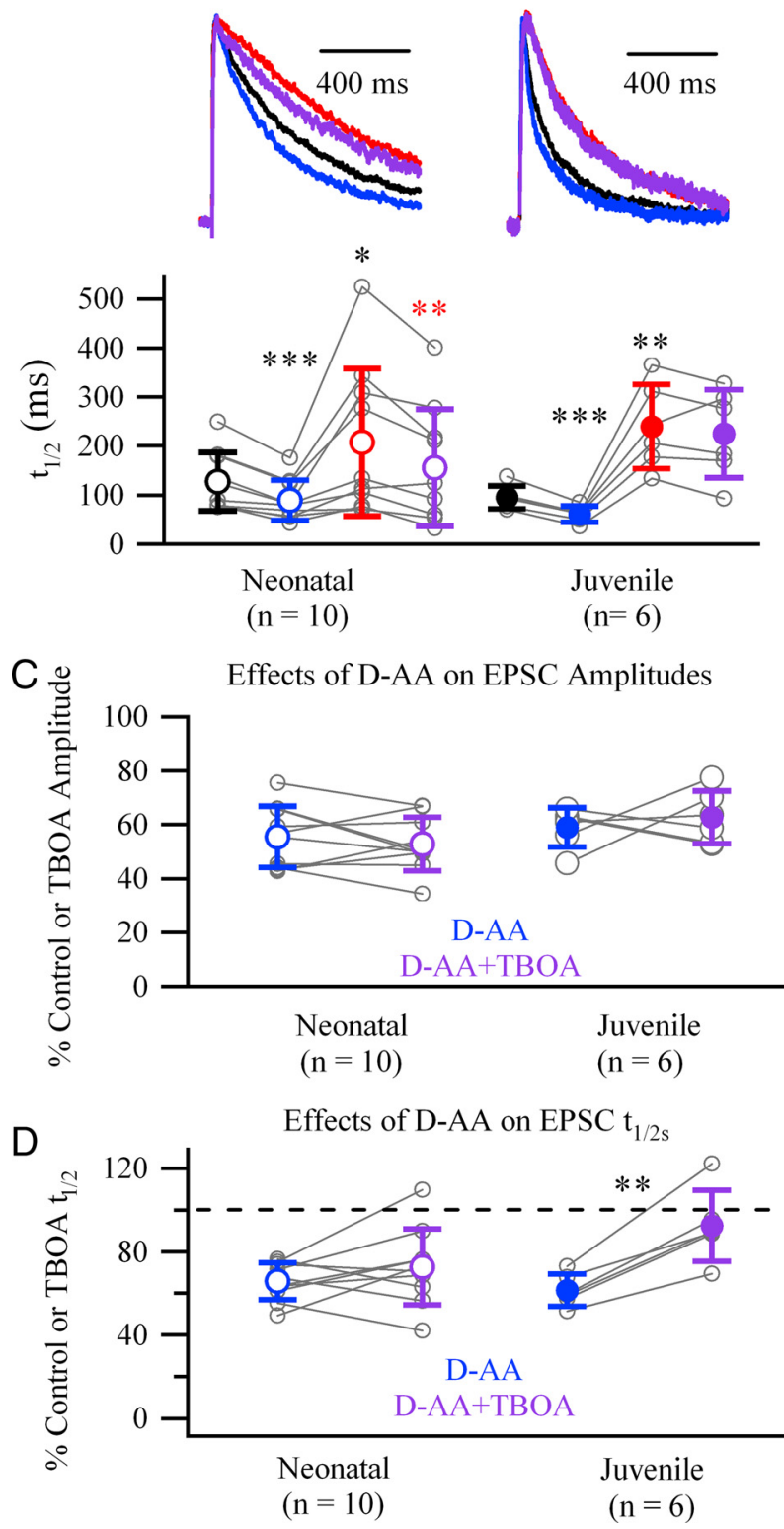

Figure 8. Regulation of extrasynaptic NMDAR activation changes during early development. $A$, EPSC amplitudes from one juvenile cell demonstrating experimental drug application sequence. $\boldsymbol{B}$, Normalized EPSCs from a neonatal cell (left side traces) or a juvenile cell (right side traces) are overlaid to demonstrate how each condition of the experiment influenced EPSC decay times. Below are graphs of the corresponding individual (light gray circles) and average greater in juvenile slices (Fig. 7C). In separate experiments, the decay times of juvenile NMDAR EPSCs remained more sensitive to TBOA even after pretreatment with the NR2B-selective antagonist, ifenprodil (5 $\mu \mathrm{M})$ (Fig. $7 D)$, indicating that developmental differences in NMDAR subunit compositions did not underlie the differences in TBOA sensitivity. Thus, extrasynaptic NMDARs in juvenile slices were more susceptible to synaptic activation than in neonatal slices, possibly because of relatively higher synaptic density and lower extracellular volume fraction in the juvenile neuropil (Fig. 2).

The relative activation of synaptic and extrasynaptic receptors can be compared using low-affinity competitive antagonists. For example, D-amino adipate (D-AA) dissociates so rapidly from NMDARs that it can be replaced by glutamate during a synaptic event (Clements et al., 1992). The extent of D-AA block therefore depends on the glutamate concentration at the receptors: NMDARs encountering large glutamate transients are blocked less than those exposed to smaller concentration waveforms (Clements et al., 1992; Diamond, 2001; Scimemi et al., 2009). Here, we compared the effects of D-AA on neonatal and juvenile NMDAR EPSCs (Fig. 8).

With glial uptake intact, D-AA $(70 \mu \mathrm{M})$ reduced NMDAR EPSC amplitudes equally in neonatal and juvenile slices (Fig. 8C), suggesting that the peak concentration of synaptically released glutamate was similar at both ages. D-AA also reduced EPSC decay times equally in neonatal and juvenile slices (Fig. $8 D$ ), suggesting that a similar fraction of NMDARs at each age was activated by smaller, slower glutamate transients and that they mediated a slower component of the EPSC that was blocked more strongly by D-AA (Diamond, 2001; Scimemi et al., 2009). In contrast, $R S$-CPP $(1 \mu \mathrm{M})$, a high-affinity antagonist that does not dissociate from NMDARs during brief synaptic events, reduced NMDAR EPSCs to a similar extent as D-AA (neonatal, $51 \pm 10 \%$ of control amplitude, $n=15$; juvenile, $54 \pm 15 \%$ of control amplitude, $n=15$ ) but had much smaller effects on EPSC decay times (neonatal, $84 \pm 13 \%$ of control $t_{1 / 2}, n=15$; juvenile, $92 \pm$ $16 \%$ of control $t_{1 / 2}, n=15$ ) (data not shown). These CPP control experiments indicated that probable improvements in space clamp occurring when EPSC amplitudes were reduced could not explain the faster decays measured in D-AA.

Next, D-AA was washed out and then applied a second time in the presence of TBOA $(30 \mu \mathrm{M})$ (Fig. $8 A$ ). Although TBOA slowed the NMDAR EPSC decay at both ages (Fig. $8 B$ ), it did not affect EPSC amplitudes or the extent to which D-AA reduced EPSC amplitudes at either age (Fig. $8 C, D$ ), suggesting that blocking uptake at either age did not affect the peak glutamate concentration within active synapses. In neonatal slices, TBOA did not change the effect of D-AA on the EPSC decay (Fig. 8D), suggesting that uptake plays a relatively small role in limiting glutamate diffusion to distant receptors. In contrast, TBOA significantly

(thick colored circles and SD bars) $t_{1 / 2}$ decay times for neonatal (left side) and juvenile (right side) EPSCs. Paired $t$ test were used to compare control versus D-AA (*** $p<0.001$ ), control versus TBOA $\left({ }^{*} p=0.03 ;{ }^{* *} p=0.002\right.$ ), and TBOA versus TBOA+DAA (red asterisks, ${ }^{* *} p=$ 0.004). C, D-AA reduces EPSC amplitudes equally for neonatal and juvenile EPSCS, regardless of whether uptake is blocked. Individual (thin gray circles) and average (thick colored circles and SD bars) neonatal (left side points) and juvenile (right side points) EPSC amplitudes recorded in D-AA and expressed as percentage control amplitudes (blue circles) or as percentage TBOA amplitudes (purple circles). $\boldsymbol{D}$, Blocking uptake changes the sensitivity of juvenile EPSCs to D-AA. D-AA decay times expressed as percentage control $t_{1 / 2}$ or as percentage TBOA $t_{1 / 2}$ for neonatal (left side) and juvenile (right side) responses. A paired $t$ test was used to compare percentage control $t_{1 / 2}$ versus percentage TBOA $t_{1 / 2}(* * p=0.001)$. 
reduced the effect of D-AA on EPSC decay in juvenile slices, suggesting that blocking uptake at this age significantly enhances the glutamate concentration at distant receptors (TBOA effect in the presence of D-AA, neonate vs juvenile, $P=0.002$ ).

\section{Discussion}

In this study, we have examined how developmental changes in glutamate uptake capacity and neuropil ultrastructure regulate synaptic activation of glutamate receptors during an early postnatal developmental period that coincides with maturation of Schaffer collateral synapses in CA1. Our results suggest that the spatial extent of NMDAR activation is similar in neonatal and juvenile slices, even though the primary regulatory mechanisms change. In neonatal slices, synaptic activation of distant NMDARs is restricted primarily by rapid dilution of glutamate into large extracellular spaces. During the second postnatal week, however, extracellular space decreases and glutamate transporter expression increases, such that uptake by transporters plays a larger role in limiting extrasynaptic NMDAR activation in juvenile slices.

Our EM results demonstrate large differences in extracellular space between neonatal and juvenile hippocampal slices. These results gave rise to the prediction, confirmed with recordings from pyramidal cells, that diffusion plays a greater role than uptake in clearing synaptically released glutamate in neonatal slices. Chemical fixation artifacts such as cell swelling may distort anatomical measurements of extracellular volume (Syková and Nicholson, 2008), possibly causing us to underestimate the in vivo extracellular volume fraction. Although less disruptive fixation methods may reduce such artifacts, our measurements agree well with values obtained by others using electron microscopy and real-time iontophoresis, and they confirm previous reports that extracellular space decreases during early postnatal development (Steward and Falk, 1991; Fiala et al., 1998; Syková and Nicholson, 2008).

\section{Activation of synaptic and extrasynaptic NMDARs during early postnatal development}

Our experimental and modeling results indicate that developmental changes in extracellular volume fraction and transporter expression regulate glutamate diffusion and NMDAR activation. In neonatal slices, glutamate is diluted more rapidly and is therefore less likely to activate extrasynaptic NMDARs. The smaller extracellular volume fraction in juvenile slices slows dilution, which would enhance extrasynaptic NMDAR activation were it not for the concomitant developmental increase in uptake capacity (Fig. 1). The low-affinity antagonist D-AA reduced the NMDAR EPSC peak to a similar extent regardless of age or whether uptake was blocked (Fig. 8). Because the early phase of the EPSC, including the peak, is likely mediated by receptors primarily located close to the site of release (Diamond, 2001), these results suggest that developmental changes in volume fraction and uptake capacity do not substantially affect NMDAR activation within active synapses. By contrast, the later component of the EPSC is mediated primarily by extrasynaptic receptors (Diamond, 2001), although late binding to synaptic receptors may also occur. Blocking uptake affected the late component more in juvenile slices (Fig. 8), indicating that glutamate transporters play a larger role later in development in limiting extrasynaptic NMDAR activation. We previously reported, however, that reducing uptake in adult slices enhances NMDAR EPSC amplitude with little effect on the decay (Diamond, 2005). Although this could indicate that glutamate transporters in the adult regu- late the activity of synaptic NMDARs exclusively, it may be that reducing uptake permits activation of extrasynaptic receptors that are activated rapidly enough to contribute to the EPSC peak. The precise location and clustering of extrasynaptic NMDARs and glutamate transporters at any developmental age is poorly understood. Our present results and previous work (Diamond, 2005) suggests that these parameters, and the consequent role of uptake in regulating NMDAR activation, continue to change throughout development into adulthood.

\section{Developmental changes in glutamate clearance at other central synapses}

Our results suggest that neuropil structure contributes substantially to the time course of neurotransmitter clearance and the role of uptake in regulating postsynaptic receptor activation. $\mathrm{Al}-$ though in CA1 developmental decreases in extracellular space slow glutamate dilution (Figs. 2, 5), the opposite transition appears to occur at mossy fiber synapses onto cerebellar granule cells, where structural changes early in development speed glutamate dilution and AMPAR-mediated EPSCs (Cathala et al., 2005). At other synapses, such as those in the supraoptic nucleus, cyclical, hormonally controlled changes in structure regulate transmitter diffusion, synaptic transmission, and plasticity (Oliet et al., 2001; Panatier et al., 2006). Hence, the relative role of neuropil structure and uptake capacity in regulating postsynaptic receptor activation appears to vary significantly at different synapse types, developmental stages, and physiological states.

\section{Regulation of transporter expression and synaptic maturation during development}

Increases in transporter expression during early postnatal development appear to compensate for the slowed dilution caused by simultaneous reductions in extracellular space. Developmental decreases in extracellular volume fraction may contribute indirectly to increased transporter expression. Neurons stimulate astrocyte transporter expression during development by releasing glutamate and soluble growth factors (Danbolt, 2001), or by making direct contact between axonal processes and astrocytes (Yang et al., 2009). Both of these processes could, in theory, be facilitated by developmental decreases in extracellular space. By contrast, contact between astrocytes and dendrites via cell adhesion molecules may have the opposite effect on transporter expression, as disrupting interactions between astrocytic ephrin-A3 ligands and dendritic EphA4 receptors increases transporter expression in CA1 (Filosa et al., 2009). Astrocyte transporter expression is enhanced during the late phase of NMDARdependent long-term potentiation in the hippocampus (PitaAlmenar et al., 2006), suggesting that glial uptake capacity is also regulated by synaptic activity and plasticity.

Similar mechanisms, such as trophic signaling, direct cell-cell contact, and synaptic plasticity, also guide synapse formation (for review, see Bourne and Harris, 2008). Although a specific role for glutamatergic signaling in synaptogenesis is unclear (MaleticSavatic et al., 1999; Lohmann and Bonhoeffer, 2008), it appears to be important for stabilization of nascent synapses (Fischer et al., 2000; Tashiro et al., 2003; Bourne and Harris, 2008) and subsequent maturation (Alvarez et al., 2007; Adesnik et al., 2008). It remains to be determined how the relationship between neuronal and glial processes, and the localization of glutamate receptors and transporters, changes during development and contributes to synaptic maturation. The results presented here suggest that multiple processes interact to regulate transporter expression at levels necessary to maintain consistent transmitter clearance, 
preserve local glutamatergic signaling, and ensure specific synaptic connectivity throughout development.

\section{References}

Adesnik H, Li G, During MJ, Pleasure SJ, Nicoll RA (2008) NMDA receptors inhibit synapse unsilencing during brain development. Proc Natl Acad Sci U S A 105:5597-5602.

Alvarez VA, Ridenour DA, Sabatini BL (2007) Distinct structural and ionotropic roles of NMDA receptors in controlling spine and synapse stability. J Neurosci 27:7365-7376.

Arnth-Jensen N, Jabaudon D, Scanziani M (2002) Cooperation between independent hippocampal synapses is controlled by glutamate uptake. Nat Neurosci 5:325-331.

Arriza JL, Fairman WA, Wadiche JI, Murdoch GH, Kavanaugh MP, Amara SG (1994) Functional comparisons of three glutamate transporter subtypes cloned from human motor cortex. J Neurosci 14:5559-5569.

Asztely F, Erdemli G, Kullmann DM (1997) Extrasynaptic glutamate spillover in the hippocampus: dependence on temperature and the role of active glutamate uptake. Neuron 18:281-293.

Barbour B (2001) An evaluation of synapse independence. J Neurosci 21:7969-7984.

Barbour B, Häusser M (1997) Intersynaptic diffusion of neurotransmitter. Trends Neurosci 20:377-384.

Bergles DE, Jahr CE (1997) Synaptic activation of glutamate transporters in hippocampal astrocytes. Neuron 19:1297-1308.

Bergles DE, Jahr CE (1998) Glial contribution to glutamate uptake at Schaffer collateral-commissural synapses in the hippocampus. J Neurosci 18:7709-7716.

Bergles DE, Tzingounis AV, Jahr CE (2002) Comparison of coupled and uncoupled currents during glutamate uptake by GLT-1 transporters. J Neurosci 22:10153-10162.

Bolshakov VY, Siegelbaum SA (1995) Regulation of hippocampal transmitter release during development and long-term potentiation. Science 269:1730-1734.

Bourne JN, Harris KM (2008) Balancing structure and function at hippocampal dendritic spines. Annu Rev Neurosci 31:47-67.

Boyer C, Schikorski T, Stevens CF (1998) Comparison of hippocampal dendritic spines in culture and in brain. J Neurosci 18:5294-5300.

Bracewell R (2000) The Fourier transform and its applications, Ed 3. Boston: McGraw-Hill.

Cathala L, Holderith NB, Nusser Z, DiGregorio DA, Cull-Candy SG (2005) Changes in synaptic structure underlie the developmental speeding of AMPA receptor-mediated EPSCs. Nat Neurosci 8:1310-1318.

Chen W, Aoki C, Mahadomrongkul V, Gruber CE, Wang GJ, Blitzblau R, Irwin N, Rosenberg PA (2002) Expression of a variant form of the glutamate transporter GLT1 in neuronal cultures and in neurons and astrocytes in the rat brain. J Neurosci 22:2142-2152.

Clements JD, Lester RA, Tong G, Jahr CE, Westbrook GL (1992) The time course of glutamate in the synaptic cleft. Science 258:1498-1501.

Cline H, Haas K (2008) The regulation of dendritic arbor development and plasticity by glutamatergic synaptic input: a review of the synaptotrophic hypothesis. J Physiol 586:1509-1517.

Cohen-Cory S (2002) The developing synapse: construction and modulation of synaptic structures and circuits. Science 298:770-776.

Danbolt NC (2001) Glutamate uptake. Prog Neurobiol 65:1-105.

Diamond JS (2001) Neuronal glutamate transporters limit activation of NMDA receptors by neurotransmitter spillover on CA1 pyramidal cells. J Neurosci 21:8328-8338.

Diamond JS (2005) Deriving the glutamate clearance time course from transporter currents in CA1 hippocampal astrocytes: transmitter uptake gets faster during development. J Neurosci 25:2906-2916.

Fiala JC, Feinberg M, Popov V, Harris KM (1998) Synaptogenesis via dendritic filopodia in developing hippocampal area CA1. J Neurosci 18:8900-8911.

Fiala JC, Kirov SA, Feinberg MD, Petrak LJ, George P, Goddard CA, Harris KM (2003) Timing of neuronal and glial ultrastructure disruption during brain slice preparation and recovery in vitro. J Comp Neurol 465:90-103.

Filosa A, Paixão S, Honsek SD, Carmona MA, Becker L, Feddersen B, Gaitanos L, Rudhard Y, Schoepfer R, Klopstock T, Kullander K, Rose CR, Pasquale EB, Klein R (2009) Neuron-glia communication via EphA4/ ephrin-A3 modulates LTP through glial glutamate transport. Nat Neurosci 12:1285-1292.
Fischer M, Kaech S, Wagner U, Brinkhaus H, Matus A (2000) Glutamate receptors regulate actin-based plasticity in dendritic spines. Nat Neurosci 3:887-894

Furuta A, Rothstein JD, Martin LJ (1997) Glutamate transporter protein subtypes are expressed differentially during rat CNS development. J Neurosci 17:8363-8375.

Hestrin S (1992) Developmental regulation of NMDA receptor-mediated synaptic currents at a central synapse. Nature 357:686-689.

Hille B (1984) Ion channels of excitable membranes, Ed 1. Sunderland, MA: Sinauer Associates.

Hsia AY, Malenka RC, Nicoll RA (1998) Development of excitatory circuitry in the hippocampus. J Neurophysiol 79:2013-2024.

Iino M, Goto K, Kakegawa W, Okado H, Sudo M, Ishiuchi S, Miwa A, Takayasu Y, Saito I, Tsuzuki K, Ozawa S (2001) Glia-synapse interaction through $\mathrm{Ca}^{2+}$-permeable AMPA receptors in bergmann glia. Science 292:926-929.

Kirov SA, Goddard CA, Harris KM (2004) Age-dependence in the homeostatic upregulation of hippocampal dendritic spine number during blocked synaptic transmission. Neuropharmacology 47:640-648.

Kirson ED, Yaari Y (1996) Synaptic NMDA receptors in developing mouse hippocampal neurones: functional properties and sensitivity to ifenprodil. J Physiol 497:437-455.

Kugler P, Schleyer V (2004) Developmental expression of glutamate transporters and glutamate dehydrogenase in astrocytes of the postnatal rat hippocampus. Hippocampus 14:975-985.

Lehre KP, Danbolt NC (1998) The number of glutamate transporter subtype molecules at glutamatergic synapses: chemical and stereological quantification in young adult rat brain. J Neurosci 18:8751-8757.

Lester RA, Jahr CE (1992) NMDA channel behavior depends on agonist affinity. J Neurosci 12:635-643.

Li Z, Sheng M (2003) Some assembly required: the development of neuronal synapses. Nat Rev Mol Cell Biol 4:833-841.

Lohmann C, Bonhoeffer T (2008) A role for local calcium signaling in rapid synaptic partner selection by dendritic filopodia. Neuron 59:253-260.

Longsworth LG (1953) Diffusion measurements, at $25^{\circ}$, of aqueous solutions of amino acids, peptides and sugars. J Am Chem Soc 75:5705-5709.

Maletic-Savatic M, Malinow R, Svoboda K (1999) Rapid dendritic morphogenesis in CAl hippocampal dendrites induced by synaptic activity. Science 283:1923-1927.

Muller D, Oliver M, Lynch G (1989) Developmental changes in synaptic properties in hippocampus of neonatal rats. Brain Res Dev Brain Res 49:105-114.

Nielsen TA, DiGregorio DA, Silver RA (2004) Modulation of glutamate mobility reveals the mechanism underlying slow-rising AMPAR EPSCs and the diffusion coefficient in the synaptic cleft. Neuron 42:757-771.

Ogata K, Kosaka T (2002) Structural and quantitative analysis of astrocytes in the mouse hippocampus. Neuroscience 113:221-233.

Oliet SH, Piet R, Poulain DA (2001) Control of glutamate clearance and synaptic efficacy by glial coverage of neurons. Science 292:923-926.

Panatier A, Gentles SJ, Bourque CW, Oliet SH (2006) Activity-dependent synaptic plasticity in the supraoptic nucleus of the rat hypothalamus. J Physiol 573:711-721.

Piet R, Vargová L, Syková E, Poulain DA, Oliet SH (2004) Physiological contribution of the astrocytic environment of neurons to intersynaptic crosstalk. Proc Natl Acad Sci U S A 101:2151-2155.

Pita-Almenar JD, Collado MS, Colbert CM, Eskin A (2006) Different mechanisms exist for the plasticity of glutamate reuptake during early longterm potentiation (LTP) and late LTP. J Neurosci 26:10461-10471.

Regan MR, Huang YH, Kim YS, Dykes-Hoberg MI, Jin L, Watkins AM, Bergles DE, Rothstein JD (2007) Variations in promoter activity reveal a differential expression and physiology of glutamate transporters by glia in the developing and mature CNS. J Neurosci 27:6607-6619.

Rusakov DA, Kullmann DM (1998) Extrasynaptic glutamate diffusion in the hippocampus: ultrastructural constraints, uptake, and receptor activation. J Neurosci 18:3158-3170.

Schmidt W, Wolf G (1988) High-affinity uptake of L- $\left[{ }^{3} \mathrm{H}\right]$ glutamate and D- $\left[{ }^{3} \mathrm{H}\right]$ aspartate during postnatal development of the hippocampal formation: a quantitative autoradiographic study. Exp Brain Res 70:50-54.

Scimemi A, Fine A, Kullmann DM, Rusakov DA (2004) NR2B-containing receptors mediate cross talk among hippocampal synapses. J Neurosci 24:4767-4777.

Scimemi A, Tian H, Diamond JS (2009) Neuronal transporters regulate glu- 
tamate clearance, NMDA receptor activation, and synaptic plasticity in the hippocampus. J Neurosci 29:14581-14595.

Shibata T, Watanabe M, Tanaka K, Wada K, Inoue Y (1996) Dynamic changes in expression of glutamate transporter mRNAs in developing brain. Neuroreport 7:705-709.

Steward O, Falk PM (1991) Selective localization of polyribosomes beneath developing synapses: a quantitative analysis of the relationships between polyribosomes and developing synapses in the hippocampus and dentate gyrus. J Comp Neurol 314:545-557.

Sutherland ML, Delaney TA, Noebels JL (1996) Glutamate transporter mRNA expression in proliferative zones of the developing and adult murine CNS. J Neurosci 16:2191-2207.

Syková E, Nicholson C (2008) Diffusion in brain extracellular space. Physiol Rev 88:1277-1340.

Tashiro A, Dunaevsky A, Blazeski R, Mason CA, Yuste R (2003) Bidirectional regulation of hippocampal mossy fiber filopodial motility by kainate receptors: a two-step model of synaptogenesis. Neuron 38:773-784.
Tzingounis AV, Wadiche JI (2007) Glutamate transporters: confining runaway excitation by shaping synaptic transmission. Nat Rev Neurosci 8:935-947.

Ullensvang K, Lehre KP, Storm-Mathisen J, Danbolt NC (1997) Differential developmental expression of the two rat brain glutamate transporter proteins GLAST and GLT. Eur J Neurosci 9:1646-1655.

Underwood E (1970) Quantitative stereology. Reading, MA: Addison-Wesley.

Wadiche JI, Tzingounis AV, Jahr CE (2006) Intrinsic kinetics determine the time course of neuronal synaptic transporter currents. Proc Natl Acad Sci U S A 103:1083-1087.

Wasling P, Hanse E, Gustafsson B (2004) Developmental changes in release properties of the CA3-CA1 glutamate synapse in rat hippocampus. J Neurophysiol 92:2714-2724.

Yang Y, Gozen O, Watkins A, Lorenzini I, Lepore A, Gao Y, Vidensky S, Brennan J, Poulsen D, Won Park J, Li Jeon N, Robinson MB, Rothstein JD (2009) Presynaptic regulation of astroglial excitatory neurotransmitter transporter GLT1. Neuron 61:880-894. 